\title{
Algèbres de Lie d'homotopie associées à une proto-bigèbre de Lie
}

\author{
Momo Bangoura
}

\begin{abstract}
Résumé. On associe à toute structure de proto-bigèbre de Lie sur un espace vectoriel $F$ de dimension finie des structures d'algèbre de Lie d'homotopie définies respectivement sur la suspension de l'algèbre extérieure de $F$ et celle de son dual $F^{*}$. Dans ces algèbres, tous les crochets $n$-aires sont nuls pour $n \geq 4$ du fait qu'ils proviennent d'une structure de proto-bigèbre de Lie. Plus généralement, on associe à un élément de degré impair de l'algèbre extérieure de la somme directe de $F$ et $F^{*}$, une collection d'applications multilinéaires antisymétriques sur l'algèbre extérieure de $F\left(\right.$ resp. $\left.F^{*}\right)$, qui vérifient les identités de Jacobi généralisées, définissant les algèbres de Lie d'homotopie, si l'élément donné est de carré nul pour le grand crochet de l'algèbre extérieure de la somme directe de $F$ et de $F^{*}$.

Abstract. To any proto-Lie algebra structure on a finite-dimensional vector space $F$, we associate homotopy Lie algebra structures defined on the suspension of the exterior algebra of $F$ and that of its dual $F^{*}$, respectively. In these algebras, all $n$-ary brackets for $n \geq 4$ vanish because the brackets are defined by the proto-Lie algebra structure. More generally, to any element of odd degree in the exterior algebra of the direct sum of $F$ and $F^{*}$, we associate a set of multilinear skew-symmetric mappings on the suspension of the exterior algebra of $F\left(\right.$ resp. $\left.F^{*}\right)$, which satisfy the generalized Jacobi identities, defining the homotopy Lie algebras, if the given element is of square zero with respect to the big bracket of the exterior algebra of the direct sum of $F$ and $F^{*}$.
\end{abstract}

\section{Introduction}

Le but de ce travail est de construire des structures d'algèbre de Lie d'homotopie sur la suspension de l'algèbre extérieure d'un espace vectoriel donné de dimension finie et sur celle de son dual, à partir de la donnée sur cet espace d'une structure de proto-bigèbre de Lie. Les algèbres de Lie d'homotopie ou $L_{\infty}$-algèbres ont été introduites par Stasheff (voir [17]) comme étant des déformations des algèbres de Lie et ont trouvé des applications en physique théorique; elles sont caractérisées par la donnée sur un espace vectoriel gradué d'une collection d'applications $n$-linéaires antisymétriques de degré $2-n$, satisfaisant un ensemble de relations généralisant l'identité de Jacobi pour les algèbres de Lie, appelées identités de Jacobi généralisées.

La notion de proto-bigèbre de Lie a été introduite par Kosmann-Schwarzbach $[8,9]$ comme une généralisation naturelle de la notion de bigèbre de Lie $[4,5]$ dont celles de quasi-bigèbre de Lie $[6,8]$ et bigèbre de quasi-Lie [8] sont des cas particuliers; elle est caractérisée par la donnée d'un espace vectoriel $F$ de dimension finie sur un corps $\mathbb{K}, \mathbb{K}=\mathbb{R}$ ou $\mathbb{C}$, et d'un élément $M$ de $\bigwedge\left(F^{*} \oplus F\right)$ de degré 1 , de carré nul par rapport à la structure d'algèbre de Lie graduée définie sur $\bigwedge\left(F^{*} \oplus F\right)$ par le grand

Reçu par la rédaction le 20 septembre, 2004 ; revu le 14 novembre, 2005.

Classification (AMS) par sujet: 17B70, 17A30.

Mots clés: algèbre de Lie d'homotopie, bigèbre de Lie, quasi-bigèbre de Lie, proto-bigèbre de Lie, crochet dérivé, jacobiateur.

(c) Société mathématique du Canada 2007. 
crochet. Ce crochet a été défini par Kostant et Sternberg [14] dans le contexte de la théorie classique de BRST, puis introduit dans la théorie des bigèbres de Lie par Lecomte et Roger [18] et utilisé par la suite par Kosmann-Schwarzbach [8,9] dans l'étude des différentes généralisations de la notion de bigèbre de Lie.

Dans [21], T. Voronov a donné une construction algébrique générale conduisant à des algèbres de Lie d'homotopie en considérant une super-algèbre de Lie $V$ avec un projecteur $P$ sur une sous-algèbre abélienne vérifant une certaine condition dite de distributivité. En fait, à partir d'un élément $\Delta$ de $V$ de degré impair, il construit une suite de crochets $n$-aires symétriques au sens $\mathbb{Z}_{2}$-gradué sur l'image de $P$, appelés crochets dérivés $[1,11,19,21,22]$ et qui vérifient les identités de Jacobi généralisées si $\Delta$ est de carré nul, c'est-à-dire définissent une structure d'algèbre de Lie d'homotopie sur l'image de $P$. La notion de crochet dérivé a été introduite dans [10] et utilisée dans la théorie des algébroïdes de Lie par Roytenberg [20].

Dans ce travail, nous nous intéressons au cas $\mathbb{Z}$-gradué, où pour un espace vectoriel $F$ de dimension finie, nous associons à toute structure de proto-bigèbre de Lie sur $F$ des structures d'algèbre de Lie d'homotopie définies respectivement sur $(\bigwedge F)[1]$ la suspension de $\bigwedge F$, et $\left(\bigwedge F^{*}\right)[1]$ celle de $\bigwedge F^{*}$; nous convenons d'appeler de telles structures des algèbres quasi-Gerstenhaber quasi-différentielles. L'intérêt de définir ces structures sur les suspensions plutôt que sur les algèbres elles-mêmes réside dans le seul fait de décalage au niveau des degrés des éléments de ces algèbres afin que les applications $n$-aires satisfassent les axiomes de la définition d'une structure d'algèbre de Lie d'homotopie.

Dans une seconde phase nous donnons la version ZZ-graduée de la construction de T. Voronov [21] dans le cas particulier de $\bigwedge\left(F^{*} \oplus F\right)$ munie du grand crochet par rapport auquel $\bigwedge F$ et $\bigwedge F^{*}$ sont des sous-algèbres abéliennes. Plus précisement, à un élément $M$ de $\bigwedge\left(F^{*} \oplus F\right)$ de degré impair quelconque, on associe des crochets $n$-aires sur $\bigwedge F$ et $\bigwedge F^{*}$ qui sont antisymétriques; un tel élément $M$ étant décomposable dans $\bigwedge\left(F^{*} \oplus F\right)$ en un nombre fini de composantes homogènes, nous donnons les expressions explicites des différents crochets en fonction de ces composantes, puis nous montrons que si le degré de $M$ est égal à $2 m+1$, alors à un facteur multiplicatif près, le crochet $n$-aire défini par la composante d'ordre $n+1($ resp. $2(2 m+3)-(n+1))$ du carré de $M$ coïncide avec le $n$-ième jacobiateur des crochets définis sur $\wedge F$ (resp. $\left.\bigwedge F^{*}\right)$. Les crochets ainsi construits vérifient les identités de Jacobi généralisées si $M$ est de carré nul et l'on obtient donc ainsi des structures d'algèbre de Lie d'homotopie $\operatorname{sur}(\bigwedge F)[1]$ et $\left(\bigwedge F^{*}\right)[1]$.

\section{Grand crochet et structures reliées}

Dans cette section nous donnons la définition du grand crochet, dont nous faisons usage pour la description sommaire de la notion de proto-bigèbre de Lie ainsi que de celles de ses principaux cas particuliers. Soit $F$ un espace vectoriel de dimension finie sur $\mathbb{K}=\mathbb{R}$ ou $\mathbb{C}$. Considérons l'algèbre extérieure de la somme directe $F^{*} \oplus F$,

$$
\bigwedge\left(F^{*} \oplus F\right)=\bigoplus_{k \geq-2} \bigwedge^{(k)}(F)
$$


où

$$
\bigwedge^{(k)}(F)=\bigoplus_{p+q=k}\left(\bigwedge^{q+1} F^{*} \otimes \bigwedge^{p+1} F\right), \quad p \geq-1, \quad q \geq-1 .
$$

Un élément de $\bigwedge^{q+1} F^{*} \otimes \bigwedge^{p+1} F$ est dit de bidegré $(p, q)$ et les éléments de $\bigwedge^{(k)}(F)$ sont dits de degré $k$. Suivant $[8,9]$ :

Définition 1 Le grand crochet est la structure d'algèbre de Lie graduée $[\cdot, \cdot]$ sur $\bigwedge\left(F^{*} \oplus F\right)$ définie par les conditions suivantes :

- si $\sigma \in \mathbb{K}$ ou $\sigma^{\prime} \in \mathbb{K},\left[\sigma, \sigma^{\prime}\right]=0$,

- si $\sigma, \sigma^{\prime} \in F,\left[\sigma, \sigma^{\prime}\right]=0$,

- si $\sigma, \sigma^{\prime} \in F^{*},\left[\sigma, \sigma^{\prime}\right]=0$,

- si $\sigma \in F$ et $\sigma^{\prime} \in F^{*},\left[\sigma, \sigma^{\prime}\right]=\left\langle\sigma^{\prime}, \sigma\right\rangle$ où $\langle\cdot, \cdot\rangle$ désigne la dualité naturelle,

- la règle de Leibniz graduée : si $\sigma \in \bigwedge^{(k)}(F), \sigma^{\prime} \in \bigwedge^{\left(k^{\prime}\right)}(F), \sigma^{\prime \prime} \in \bigwedge^{\left(k^{\prime \prime}\right)}(F)$,

$$
\left[\sigma, \sigma^{\prime} \wedge \sigma^{\prime \prime}\right]=\left[\sigma, \sigma^{\prime}\right] \wedge \sigma^{\prime \prime}+(-1)^{k k^{\prime}} \sigma^{\prime} \wedge\left[\sigma, \sigma^{\prime \prime}\right] .
$$

Comme structure d'algèbre de Lie graduée, le grand crochet vérifie les propriétés suivantes :

- l'antisymétrie graduée

$$
\left[\sigma, \sigma^{\prime}\right]=-(-1)^{k k^{\prime}}\left[\sigma^{\prime}, \sigma\right]
$$

si $\sigma \in \bigwedge^{(k)}(F), \sigma^{\prime} \in \bigwedge^{\left(k^{\prime}\right)}(F)$,

- l'identité de Jacobi graduée

$$
\oint(-1)^{k_{1} k_{3}}\left[\sigma_{1},\left[\sigma_{2}, \sigma_{3}\right]\right]=0
$$

si $\sigma_{1} \in \bigwedge^{\left(k_{1}\right)}(F), \sigma_{2} \in \bigwedge^{\left(k_{2}\right)}(F), \sigma_{3} \in \bigwedge^{\left(k_{3}\right)}(F)$, où $\oint$ désigne la somme sur les permutations circulaires de $\{1,2,3\}$.

Remarque $\bigwedge F$ et $\bigwedge F^{*}$ sont des sous-algèbres de Lie abéliennes de $\left(\bigwedge\left(F^{*} \oplus F\right)\right.$, $[\cdot, \cdot])$. Dans tout ce qui va suivre le grand crochet constituera l'outil fondamental de travail avec l'usage dans la démonstration des principaux résultats des deux propriétés ci-dessus énoncées.

Suivant [8] on a la définition suivante.

Définition 2 Un élément de degré $1, M \in \bigwedge^{(1)}(F)$, est une structure de protobigèbre de Lie sur $F$ s'il est de carré nul dans $\left(\bigwedge\left(F^{*} \oplus F\right),[\cdot, \cdot]\right)$, i.e., $[M, M]=0$.

Un tel élément est de la forme $M=\mu+\gamma+\varphi+\psi$, où $\mu \in \bigwedge^{2} F^{*} \otimes F, \gamma \in F^{*} \otimes \bigwedge^{2} F$, $\varphi \in \Lambda^{3} F$ et $\psi \in \Lambda^{3} F^{*}$. Tenant compte des bidegrés des différents éléments, la condition $[M, M]=0$ est équivalente aux suivantes:

$$
\begin{gathered}
\frac{1}{2}[\mu, \mu]+[\gamma, \psi]=0, \quad[\mu, \gamma]+[\varphi, \psi]=0, \quad \frac{1}{2}[\gamma, \gamma]+[\mu, \varphi]=0, \\
{[\mu, \psi]=0, \quad[\gamma, \varphi]=0 .}
\end{gathered}
$$


Ce qui nous conduit à la définition équivalente suivante.

Définition 3 Pour un espace vectoriel donné $F$ et des éléments $\mu, \gamma, \varphi$ et $\psi$ donnés de $\bigwedge\left(F^{*} \oplus F\right)$ satisfaisant les conditions ci-dessus, $(F, \mu, \gamma, \varphi, \psi)$ est appelé une protobigèbre de Lie.

\section{Remarques}

1. Dans [3] et [18], un tel élément de degré 1 de carré nul est appelé simplement une structure.

2. Si $(F, \mu, \gamma, \varphi, \psi)$ est une proto-bigèbre de Lie, alors $\left(F^{*}, \gamma, \mu, \psi, \varphi\right)$ est aussi une proto-bigèbre de Lie, ainsi la notion de proto-bigèbre de Lie est auto-duale.

Définition 4 Si $\psi=0$ (resp. $\varphi=0)$ le quadruplet $(F, \mu, \gamma, \varphi)(\operatorname{resp} .(F, \mu, \gamma, \psi))$ satisfaisant les conditions ci-dessus est appelé une quasi-bigèbre de Lie (resp. bigèbre de quasi-Lie).

On remarque que ces deux notions sont duales l'une de l'autre et ne sont pas autoduales. La notion de quasi-bigèbre de Lie est due à Drinfeld [6]. Si $\varphi=\psi=0$, le triplet $(F, \mu, \gamma)$ est une bigèbre de Lie $[4,5]$; cette dernière notion comme celle de proto-bigèbre de Lie est auto-duale.

Exemple 1 Soit $(F, \mu)$ une algèbre de Lie. Alors tout choix d'un élément $\mathbf{r} \in \bigwedge^{2} F$ définit une structure de quasi-bigèbre de Lie sur $F$ en posant

$$
\gamma=[\mu, \mathbf{r}], \quad \varphi=\frac{1}{2}[\mathbf{r}, \mathbf{r}]^{\mu}
$$

où $[\mathbf{r}, \mathbf{r}]^{\mu}$ désigne le crochet de Schouten algébrique $[13,15]$ de $\mathbf{r}$ avec lui-même.

Exemple 2 Soit $F$ un espace vectoriel de dimension finie; soient $\varphi \in \bigwedge^{3} F$ et $\omega \in$ $\bigwedge^{2} F^{*}$. Posons

$$
\mu=\frac{1}{2}[\omega,[\omega, \varphi]], \quad \gamma=[\omega, \varphi], \quad \psi=\frac{1}{6}[\omega,[\omega,[\omega, \varphi]]]
$$

Alors $(F, \mu, \gamma, \varphi, \psi)$ est une proto-bigèbre de Lie.

Exemple 3 Soit $F$ un espace vectoriel de dimension finie; soient $\mathbf{r} \in \bigwedge^{2} F$ et $\psi \in$ $\bigwedge^{3} F^{*}$. Posons

$$
\mu=[\mathbf{r}, \psi], \quad \gamma=\frac{1}{2}[\mathbf{r},[\mathbf{r}, \psi]], \quad \varphi=\frac{1}{6}[\mathbf{r},[\mathbf{r},[\mathbf{r}, \psi]]] .
$$

Alors $(F, \mu, \gamma, \varphi, \psi)$ est une proto-bigèbre de Lie. 


\section{Algèbres de Lie d'homotopie}

De $[16,17,21]$ on a la définition suivante.

Définition 5 Une algèbre de Lie d'homotopie ou $L_{\infty}$-algèbre est un espace vectoriel gradué $V$ muni d'une collection d'applications $n$-linéaires antisymétriques

$$
l_{n}: \bigotimes^{n} V \mapsto V
$$

de degré $2-n$, telle que

$$
\sum_{i+j=n+1} \sum_{\sigma} \operatorname{sign}(\sigma) \varepsilon(\sigma)(-1)^{i(j-1)} l_{i}\left(l_{j}\left(x_{\sigma(1)}, \ldots, x_{\sigma(j)}\right), x_{\sigma(j+1)}, \ldots, x_{\sigma(n)}\right)=0,
$$

pour tout $n=0,1, \ldots$ où $\sigma$ est un " $(j, n-j)$-shuffle", c'est-à-dire une permutation des indices $1, \ldots, n$ telle que $\sigma(1)<\cdots<\sigma(j)$ et $\sigma(j+1)<\cdots<\sigma(n), \operatorname{sign}(\sigma)$ désigne la signature de $\sigma$ et $\varepsilon(\sigma)$ est le signe de Koszul défini par

$$
x_{1} \wedge x_{2} \wedge \cdots \wedge x_{n}=\varepsilon(\sigma) x_{\sigma(1)} \wedge x_{\sigma(2)} \wedge \cdots \wedge x_{\sigma(n)} .
$$

Les identités ci-dessus, définissant une structure de $L_{\infty}$-algèbre, sont appélées les identités de Jacobi généralisées et le membre de gauche de ces égalités pour chaque $n$ est appelé le n-ième jacobiateur.

Remarque Dans [17], le cas $n=0$ ne figure pas dans la définition d'une $L_{\infty}$-algèbre; les structures de $L_{\infty}$-algèbre avec $\phi=0$ sont appelées strictes dans [21]. Les $L_{\infty}$ algèbres avec $\phi \neq 0$ ont été appelées faibles ou "with background" par certains physiciens. Ici et dans toute la suite, une application 0 -aire signifiera simplement un élément distingué $\phi$ de $V$ correspondant à l'image de l'élément unité 1 de $\mathbb{K}$, i.e., $\phi=l_{0}(1)$.

Les identités de Jacobi généralisées s'écrivent explicitement pour $n=0,1$ et 2 sous la forme:

$$
\begin{array}{cc}
(n=0) & l_{1}\left(l_{0}(1)\right)=l_{1}(\phi)=0, \\
(n=1) & l_{1}\left(l_{1}(x)\right)+l_{2}(\phi, x)=0, \\
(n=2) & -l_{1}\left(l_{2}(x, y)\right)+l_{2}\left(l_{1}(x), y\right)-(-1)^{|x||y|} l_{2}\left(l_{1}(y), x\right)-l_{3}(\phi, x, y)=0,
\end{array}
$$

pour tous $x, y \in V$.

Dans le cas strict, les identités pour $n=1$ et $n=2$ expriment que $l_{1}$ est de carré nul et est une dérivation de degré 1 par rapport à $l_{2}$.

\section{Crochets $n$-aires associés à une structure de proto-bigèbre de Lie}

Soit $M=\mu+\gamma+\varphi+\psi \in \bigwedge\left(F^{*} \oplus F\right)$ une structure de proto-bigèbre de Lie sur $F$ comme définie dans la section 2 . Sur $\bigwedge F$ (resp. $\bigwedge F^{*}$ ), définissons des applications 
$n$-aires $\{\cdot, \ldots, \cdot\}: \bigotimes^{n} \wedge F \mapsto \bigwedge F\left(\right.$ resp. $\left.\{\cdot, \ldots, \cdot\}^{*}: \bigotimes^{n} \wedge F^{*} \mapsto \bigwedge F^{*}\right)$ de la manière suivante :

$$
\begin{gathered}
\{1\}=\varphi, \quad\left\{x_{1}\right\}=\left[\gamma, x_{1}\right], \quad\left\{x_{1}, x_{2}\right\}=(-1)^{\left(\left|x_{1}\right|-1\right)}\left[\left[\mu, x_{1}\right], x_{2}\right]=\left[x_{1}, x_{2}\right]^{\mu}, \\
\left\{x_{1}, x_{2}, x_{3}\right\}=(-1)^{\left(\left|x_{2}\right|-1\right)}\left[\left[\left[\psi, x_{1}\right], x_{2}\right], x_{3}\right], \\
\left\{x_{1}, \ldots, x_{n}\right\}=0 \text { pour } n \geq 4
\end{gathered}
$$

respectivement,

$$
\begin{gathered}
\{1\}^{*}=\psi, \quad\left\{\alpha_{1}\right\}^{*}=\left[\mu, \alpha_{1}\right], \\
\left\{\alpha_{1}, \alpha_{2}\right\}^{*}=(-1)^{\left(\left|\alpha_{1}\right|-1\right)}\left[\left[\gamma, \alpha_{1}\right], \alpha_{2}\right]=\left[\alpha_{1}, \alpha_{2}\right]^{\gamma}, \\
\left\{\alpha_{1}, \alpha_{2}, \alpha_{3}\right\}^{*}=(-1)^{\left(\left|\alpha_{2}\right|-1\right)}\left[\left[\left[\varphi, \alpha_{1}\right], \alpha_{2}\right], \alpha_{3}\right], \\
\left\{\alpha_{1}, \ldots, \alpha_{n}\right\}^{*}=0 \quad \text { pour } n \geq 4 .
\end{gathered}
$$

Dans $[1,21,22]$, de telles applications $n$-aires sont obtenues par applications successives de la construction de crochets dérivés. Elles sont dites crochets dérivés d'ordre supérieur. Ici nous les appellerons les crochets $n$-aires associés à la structure $M$. Le fait que les crochets $n$-aires soient identiquement nuls pour $n \geq 4$ est une conséquence logique de la décomposition de $M$ en quatre composantes homogènes, car chaque crochet dérive d'une seule composante de $M$.

Soit $(\bigwedge F)[1]$ (resp., $\left.\left(\bigwedge F^{*}\right)[1]\right)$ la suspension de $\bigwedge F$ (resp., $\left.\bigwedge F^{*}\right)$, i.e., $\left(\bigwedge^{p} F\right)[1]=\bigwedge^{p+1} F\left(\right.$ resp.,$\left.\left(\bigwedge^{p} F^{*}\right)[1]=\bigwedge^{p+1} F^{*}\right)$.

Remarques $\varphi \in \bigwedge^{3} F$ est de degré 2 dans $\bigwedge F[1]$; le degré du crochet 0 -aire \{\} est donc égal à 2 dans $(\bigwedge F)[1]$, alors qu'il est de degré 3 dans $\bigwedge F$.

Par ailleurs, pour $x_{1} \in \bigwedge^{p+1} F$ on a $\left[\gamma, x_{1}\right] \in \bigwedge^{p+2} F$; le degré du crochet unaire $\{\cdot\}$ est ainsi égal à 1 .

Pour $x_{1} \in \bigwedge^{p+1} F, x_{2} \in \bigwedge^{q+1} F$, on a $\left[\left[\mu, x_{1}\right], x_{2}\right] \in \bigwedge^{p+q+1} F$; par conséquent le degré du crochet binaire $\{\cdot, \cdot\}$ dans $(\bigwedge F)[1]$ est égal à 0 alors qu'il est de degré -1 dans $\bigwedge F$.

Pour $x_{1} \in \bigwedge^{p+1} F, x_{2} \in \bigwedge^{q+1} F, x_{3} \in \bigwedge^{r+1} F$, on a $\left[\left[\left[\psi, x_{1}\right], x_{2}\right], x_{3}\right] \in \bigwedge^{p+q+r} F$; donc le degré du crochet trilinéaire $\{\cdot, \cdot, \cdot\}$ dans $(\bigwedge F)[1]$ est égal à -1 , alors qu'il est de degré -3 dans $\bigwedge F$.

Ainsi comme on le voit, le degré du crochet $n$-aire est égal à $(2-n)$, pour tout $n$. Les mêmes remarques sont valables pour les crochets définis sur $\bigwedge F^{*}$.

On a le résultat suivant.

Proposition 1 Soit $M=\mu+\gamma+\varphi+\psi$ une structure de proto-bigèbre de Lie sur F. Alors les crochets $n$-aires $\{\cdot, \ldots, \cdot\}$ (resp. $\left.\{\cdot, \ldots, \cdot\}^{*}\right)$ associés à $M$ et définis sur $(\bigwedge F)[1]\left(\right.$ resp. $\left.\left(\bigwedge F^{*}\right)[1]\right)$ sont antisymétriques, de degré $2-n$. De plus chaque crochet $n$-aire est une dérivation en chacun de ses arguments. 
Démonstration On démontre la proposition dans le cas de $(\bigwedge F)[1])$, le cas $\left(\bigwedge F^{*}\right)[1]$ étant analogue. L'antisymétrie des crochets $n$-aires est une conséquence directe de l'identité de Jacobi graduée pour le grand crochet; on a

$$
\left\{x_{1}, x_{2}\right\}=-(-1)^{\left(\left|x_{1}\right|-1\right)\left(\left|x_{2}\right|-1\right)}\left\{x_{2}, x_{1}\right\}
$$

et

$$
\begin{aligned}
\left\{x_{1}, x_{2}, x_{3}\right\} & =-(-1)^{\left(\left|x_{1}\right|-1\right)\left(\left|x_{2}\right|-1\right)}\left\{x_{2}, x_{1}, x_{3}\right\} \\
& =-(-1)^{\left(\left|x_{2}\right|-1\right)\left(\left|x_{3}\right|-1\right)}\left\{x_{1}, x_{3}, x_{2}\right\} .
\end{aligned}
$$

La condition de degré $(2-n)$ est satisfaite grâce aux remarques précédentes. Ce qui démontre la proposition.

Remarque Les crochets $n$-aires ci-dessus définis, bien qu'étant antisymétriques sur $(\bigwedge F)[1]$, ne le sont pas sur $\bigwedge F$ comme le montrent les relations ci-dessus.

On peut à présent lier les crochets $n$-aires associés à une structure de proto-bigèbre de Lie $M$ sur $F$ avec la donnée d'une structure d'algèbre de Lie d'homotopie; plus précisement on a :

Théorème 1 Soit $M=\mu+\gamma+\varphi+\psi \in \bigwedge\left(F^{*} \oplus F\right)$ une structure de proto-bigèbre de Lie sur $F$; alors les crochets $n$-aires $l_{n}=\{\cdot, \ldots, \cdot\}$ (resp. $\left.l_{n}^{*}=\{\cdot, \ldots, \cdot\}^{*}\right)$ sur $\bigwedge F$ (resp. $\left.\bigwedge F^{*}\right)$ associés à $M$ définissent une structure de $L_{\infty}$-algèbre sur $(\bigwedge F)[1]$ (resp. $\left.\left(\bigwedge F^{*}\right)[1]\right)$.

Démonstration Démontrons le résultat dans le cas de $(\bigwedge F)[1]$, le cas de $\left(\bigwedge F^{*}\right)[1]$ est analogue; d'après la proposition précédente, chaque crochet $n$-aire associé à $M$ est antisymétrique et de degré $2-n$. Les identités de Jacobi généralisées sont des conséquences directes de la condition $[M, M]=0$ et de l'identité de Jacobi graduée pour le grand crochet. Comme $\{\cdot, \ldots, \cdot\}=0$ pour $n \geq 4$, nous avons à démontrer les identités de Jacobi généralisées pour $n=0,1,2,3,4$ et 5 . En effet

- pour $n=0$, on a:

$$
\{\{1\}\}=\{\varphi\}=[\gamma, \varphi]=0,
$$

d'après $[M, M]=0$; ce qui démontre l'identité de Jacobi généralisée pour $n=0$.

- Pour $n=1, x_{1} \in \bigwedge F$ on a :

$$
\left\{\left\{x_{1}\right\}\right\}=\left[\gamma,\left[\gamma, x_{1}\right]\right]=\frac{1}{2}\left[[\gamma, \gamma], x_{1}\right]=-\left[[\mu, \varphi], x_{1}\right]=-\left\{\varphi, x_{1}\right\}
$$

i.e.,

$$
\left\{\left\{x_{1}\right\}\right\}+\left\{\varphi, x_{1}\right\}=0
$$

ce qui démontre l'identité de Jacobi généralisée pour $n=1$. 
- Pour $n=2, x_{1} \in \bigwedge^{p} F, x_{2} \in \bigwedge^{q} F$, utilisant l'identité de Jacobi graduée pour le grand crochet on a :

$$
\begin{aligned}
\left\{\left\{x_{1}, x_{2}\right\}\right\}= & {\left[\gamma,\left[\left[x_{1}, \mu\right], x_{2}\right]\right] } \\
= & {\left[\left[\left[\gamma, x_{1}\right], \mu\right], x_{2}\right]+(-1)^{p}\left[\left[x_{1},[\gamma, \mu]\right], x_{2}\right] } \\
& +(-1)^{p-1}\left[\left[x_{1}, \mu\right],\left[\gamma, x_{2}\right]\right] \\
= & \left\{\left\{x_{1}\right\}, x_{2}\right\}+(-1)^{p-1}\left\{x_{1},\left\{x_{2}\right\}\right\}-(-1)^{p}\left[\left[x_{1},[\varphi, \psi]\right], x_{2}\right] \\
= & \left\{\left\{x_{1}\right\}, x_{2}\right\}+(-1)^{p-1}\left\{x_{1},\left\{x_{2}\right\}\right\}-(-1)^{(p-1)}\left[\left[[\psi, \varphi], x_{1}\right], x_{2}\right] \\
= & \left\{\left\{x_{1}\right\}, x_{2}\right\}+(-1)^{p-1}\left\{x_{1},\left\{x_{2}\right\}\right\}-\left\{\varphi, x_{1}, x_{2}\right\} ;
\end{aligned}
$$

d'où

$$
\left\{\left\{x_{1}, x_{2}\right\}\right\}-\left\{\left\{x_{1}\right\}, x_{2}\right\}+(-1)^{(p-1)(q-1)}\left\{\left\{x_{2}\right\}, x_{1}\right\}+\left\{\varphi, x_{1}, x_{2}\right\}=0
$$

ce qui prouve l'identité de Jacobi généralisée pour $n=2$.

- Les cas $n=3,4$ et 5 s'obtiennent respectivement des conditions $\frac{1}{2}[\mu, \mu]+[\gamma, \psi]=$ $0,[\mu, \psi]=0$ et $[\psi, \psi]=0$ de la définition d'une structure de proto-bigèbre de Lie, en utilisant l'identité de Jacobi graduée pour le grand crochet.

- Pour $n \geq 6$, l'identité de Jacobi généralisée est une trivialité parce que $\{\cdot, \ldots, \cdot\}$ $=0$ pour $k \geq 4$. Ce qui démontre le théorème.

Ainsi on vient de prouver qu'à toute structure de proto-bigèbre de Lie $(F, \mu, \gamma, \varphi, \psi)$ sur un espace vectoriel $F$ de dimension finie, correspondent deux structures de $L_{\infty}$-algèbre définies respectivement sur $(\bigwedge F)[1]$ et $\left(\bigwedge F^{*}\right)[1]$ dont les éléments distingués sont respectivement $\varphi$ et $\psi$. Nous appellerons de telles structures des algèbres quasi-Gerstenhaber quasi-différentielles. Si $\varphi=0$ (resp. $\psi=0$ ), on obtient une structure d'algèbre quasi-Gerstenhaber différentielle (resp. d'algèbre de Gerstenhaber quasi-différentielle) $[2,12,20]$. Huebschmann a défini dans [7] une notion d'algèbre quasi-Gerstenhaber différentielle bigraduée qu'il compare à la nôtre. Comme conséquence directe du théorème précédent, on a :

\section{Corollaire 1}

(1) A toute structure de quasi-bigèbre de Lie $(F, \mu, \gamma, \varphi)$ sur un espace vectoriel $F$ de dimension finie, il correspond une structure d'algèbre de Gerstenhaber quasi-différentielle (resp. d'algèbre quasi-Gerstenhaber différentielle) sur $(\bigwedge F)[1]$ (resp. $\left.\left(\bigwedge F^{*}\right)[1]\right)$.

(2) A toute structure de bigèbre de quasi-Lie $(F, \mu, \gamma, \psi)$ sur un espace vectoriel $F$ de dimension finie, il correspond une structure d'algèbre quasi-Gerstenhaber différentielle (resp. d'algèbre de Gerstenhaber quasi-différentielle) sur $(\bigwedge F)[1]$ (resp. $\left.\left(\bigwedge F^{*}\right)[1]\right)$.

Remarque Les structures d'algèbre quasi-Gerstenhaber différentielle obtenues sur $\left(\bigwedge F^{*}\right)[1]$ en prenant $\psi=0$ sont celles étudiées dans [2], ce sont des structures de $L_{\infty}$-algèbre strictes au sens de [21]. 
Cas des algébroïdes de Lie En utilisant la généralisation du grand crochet introduite par Roytenberg [20], les démonstrations ci-dessus s'étendent au cas des algébroïdes de Lie. Donc plus généralement, à toute structure de proto-bigébroïde de Lie donnée $\left(\left(A, A^{*}\right), \mu, \gamma, \varphi, \psi\right)[12,20]$, il correspond deux structures d'algèbre quasi-Gerstenhaber quasi-différentielle définies respectivement sur $(\Gamma(\bigwedge A))[1]$ et $\left(\Gamma\left(\bigwedge A^{*}\right)\right)[1]$, où les différents crochets $n$-aires sont définis de manière analogue en fonction de $\mu, \gamma, \varphi$ et $\psi$ comme dans le cas d'une proto-bigèbre de Lie.

\section{Crochets $n$-aires associés à un élément de degré impair de $\bigwedge\left(F^{*} \oplus F\right)$}

Dans cette section, nous donnons une généralisation de la construction de structures de $L_{\infty}$-algèbre sur $(\bigwedge F)[1]$ et $\left(\bigwedge F^{*}\right)[1]$ donnée dans la section précédente.

Remplaçant la condition de degré $(2-n)$ par $(2(m+1)-n)$ pour un $m$ fixé dans la définition d'une $L_{\infty}$-algèbre tout en gardant l'antisymétrie graduée et les identités de Jacobi généralisées, on peut construire des opérations $n$-aires sur $\bigwedge F$ et $\bigwedge F^{*}$ par le choix d'un élément $M \in \bigwedge\left(F^{*} \oplus F\right)$ de degré impair.

\section{Remarques}

1. Pour $m=0$, nous retrouvons la condition de degré $(2-n)$ de la définition d'une $L_{\infty}$-algèbre.

2. Pour un élément $M$ de degré pair, on a automatiquement $[M, M]=0$, mais les crochets dérivés qu'il engendre ne satisfont pas aux conditions de la généralisation souhaitée notamment la condition de degré; ce cas est donc exclu de la présente démarche.

Soit $M \in \bigwedge\left(F^{*} \oplus F\right)$ tel que $|M|=2 m+1$; alors $M$ est de la forme

$$
M=\sum_{q=0}^{2 m+3} M_{q},
$$

où $M_{q} \in \bigwedge^{q} F^{*} \otimes \bigwedge^{2 m+3-q} F$. Tenant compte des bidegrés, $[M, M]$ s'écrit sous la forme

$$
[M, M]=\sum_{k=0}^{2(2 m+3)} \sum_{p+q=k}\left[M_{p}, M_{q}\right] .
$$

Posons

$$
M^{(k)}=\sum_{p+q=k}\left[M_{p}, M_{q}\right]
$$

et appelons $M^{(k)}$ la composante d'ordre $k$ du carré de $M$. Ainsi $M$ est de carré nul si et seulement si

$$
M^{(k)}=\sum_{p+q=k}\left[M_{p}, M_{q}\right]=0
$$

pour tous $k=0,1, \ldots, 2(2 m+3)$. Si $m=0, M$ est de degré 1 et nous retrouvons les conditions définissant une structure de proto-bigèbre de Lie sur $F$ avec $M_{0}=\varphi$, $M_{1}=\gamma, M_{2}=\mu, M_{3}=\psi$. 
Lemme 1 Pour tout élément $M=\sum_{q=0}^{2 m+3} M_{q} \in \bigwedge^{(2 m+1)}(F)$, pour $x_{1}, x_{2}, \ldots, x_{n} \in$ $\bigwedge F, \alpha_{1}, \alpha_{2}, \ldots, \alpha_{n} \in \bigwedge F^{*}$ on $a:$

(i) $\left[\left[\left[\ldots\left[M_{p}, x_{1}\right], x_{2}\right], \ldots\right], x_{n}\right] \in \wedge F$ si et seulement si $p=n$;

(ii) $\left[\left[\left[\ldots\left[M_{p}, \alpha_{1}\right], \alpha_{2}\right], \ldots\right], \alpha_{n}\right] \in \wedge F^{*}$ si et seulement si $p=2 m+3-n$;

(iii) $\left[\left[\left[\ldots\left[M^{(k)}, x_{1}\right], x_{2}\right], \ldots\right], x_{n}\right] \in \bigwedge F$ si et seulement si $k=n+1$;

(iv) $\left[\left[\left[\ldots\left[M^{(k)}, \alpha_{1}\right], \alpha_{2}\right], \ldots\right], \alpha_{n}\right] \in \bigwedge F^{*}$ si et seulement si $k=2(2 m+3)-(n+1)$.

Démonstration Montrons (i) et (iii), (ii) et (iv) se démontrant de manière similaire. Par définition du grand crochet, on a :

(i)

$$
\left[\left[\left[\ldots\left[M_{p}, x_{1}\right], x_{2}\right], \ldots\right], x_{n}\right] \in \bigwedge^{p-n} F^{*} \otimes \bigwedge^{S} F,
$$

où $S=2 m+3-p-n+\sum_{i=1}^{n}\left|x_{i}\right|$; d'où $\left[\left[\left[\ldots\left[M_{p}, x_{1}\right], x_{2}\right], \ldots\right], x_{n}\right] \in \wedge F$ si et seulement si $p=n$.

(iii)

$$
\left[\left[\left[\ldots\left[M^{(k)}, x_{1}\right], x_{2}\right], \ldots\right], x_{n}\right]=\sum_{p+q=k}\left[\left[\left[\ldots\left[\left[M_{p}, M_{q}\right], x_{1}\right], x_{2}\right], \ldots\right], x_{n}\right] ;
$$

mais

$$
\left[\left[\left[\ldots\left[\left[M_{p}, M_{q}\right], x_{1}\right], x_{2}\right], \ldots\right], x_{n}\right] \in \bigwedge^{p+q-(n+1)} F^{*} \otimes \bigwedge^{T} F
$$

où $T=\left(4 m+5-(p+q)-n+\sum_{i=1}^{n}\left|x_{i}\right|\right) ;$ d'où $\left[\left[\left[\ldots\left[M^{(k)}, x_{1}\right], x_{2}\right], \ldots\right], x_{n}\right] \in \bigwedge F$ si et seulement si $k=p+q=n+1$. Ce qui achève la démonstration du lemme.

Désignons par $\prod_{\bigwedge F}$ (resp. $\prod_{\bigwedge F^{*}}$ ) la projection de $\bigwedge\left(F^{*} \oplus F\right)$ sur $\bigwedge F$ (resp. $\left.\bigwedge F^{*}\right)$. Associons à $M$ des crochets $n$-aires sur $\Lambda F$ de la manière suivante :

$$
\begin{gathered}
\{1\}_{M}=\prod_{\wedge F} M, \\
\left\{x_{1}, \ldots, x_{n}\right\}_{M}=(-1)^{\theta_{n}} \prod_{\wedge F}\left[\left[\ldots\left[M, x_{1}\right], \ldots\right], x_{n}\right],
\end{gathered}
$$

où $x_{1}, \ldots, x_{n} \in \bigwedge F, \theta_{n}=\sum_{k=1}^{n-1}(n-k)\left(\left|x_{k}\right|-1\right)$; et sur $\bigwedge F^{*}$ de la manière suivante :

$$
\begin{gathered}
\{1\}_{M}^{*}=\prod_{\bigwedge F^{*}} M, \\
\left\{\alpha_{1}, \ldots, \alpha_{n}\right\}_{M}^{*}=(-1)^{\theta_{n}^{*}} \prod_{\bigwedge F^{*}}\left[\left[\ldots\left[M, \alpha_{1}\right], \ldots\right], \alpha_{n}\right],
\end{gathered}
$$

où $\alpha_{1}, \ldots, \alpha_{n} \in \bigwedge F^{*}, \theta_{n}^{*}=\sum_{k=1}^{n-1}(n-k)\left(\left|\alpha_{k}\right|-1\right)$.

Le résultat suivant donne les expressions explicites des différents crochets en fonction des composantes $M_{q}$ de $M$ : 
Proposition 2 Soit $M=\sum_{q=0}^{2 m+3} M_{q} \in \bigwedge^{(2 m+1)}(F)$ où $M_{q} \in \bigwedge^{q} F^{*} \otimes \bigwedge^{2 m+3-q} F$; alors les crochets $n$-aires $\{\cdot, \ldots, \cdot\}_{M}$ et $\{\cdot, \ldots, \cdot\}_{M}^{*}$ associés à $M$ sont antisymétriques, de degré $2(m+1)-n$ sur $(\bigwedge F)[1]$ et $\left(\bigwedge F^{*}\right)[1]$ respectivement, et satisfont:

$$
\begin{gathered}
\{1\}_{M}=M_{0}, \\
\left\{x_{1}, \ldots, x_{n}\right\}_{M}=(-1)^{\theta_{n}}\left[\left[\ldots\left[M_{n}, x_{1}\right], \ldots\right], x_{n}\right],
\end{gathered}
$$

$x_{1}, \ldots, x_{n} \in \bigwedge F$, où $\theta_{n}=\sum_{k=1}^{n-1}(n-k)\left(\left|x_{k}\right|-1\right)$,

$$
\begin{gathered}
\{1\}_{M}^{*}=M_{2 m+3}, \\
\left\{\alpha_{1}, \ldots, \alpha_{n}\right\}_{M}^{*}=(-1)^{\theta_{n}^{*}}\left[\left[\ldots\left[M_{2 m+3-n}, \alpha_{1}\right], \ldots\right], \alpha_{n}\right],
\end{gathered}
$$

$\alpha_{1}, \ldots, \alpha_{n} \in \bigwedge F^{*}$, oй $\theta_{n}^{*}=\sum_{k=1}^{n-1}(n-k)\left(\left|\alpha_{k}\right|-1\right)$.

Ce résultat suit par l'identité de Jacobi gradué et le lemme précédent.

\section{Remarques}

1. Ces crochets associés à $M$ sont antisymétriques et satisfont la condition de degré $2(m+1)-n \operatorname{sur}(\bigwedge F)[1]$ et $\left(\bigwedge F^{*}\right)[1]$, mais pas sur $\bigwedge F$ et $\bigwedge F^{*}$ à cause du décalage au niveau des degrés; d'où l'intérêt de travailler avec $(\bigwedge F)[1]$ et $\left(\bigwedge F^{*}\right)[1]$.

2. $\left\{x_{1}, \ldots, x_{n}\right\}_{M}=0$ et $\left\{\alpha_{1}, \ldots, \alpha_{n}\right\}_{M}^{*}=0$ pour $n>2 m+3$, sont des conséquences de la décomposition de $M$ en nombre fini de composantes homogènes.

Lemme 2 Soit $M \in \bigwedge^{(2 m+1)}(F) ;$ si $p+q=n+1$ alors pour $x_{1}, \ldots, x_{n} \in \bigwedge F$, on a

$$
\begin{aligned}
& {\left[\left[\ldots\left[\left[M_{p}, M_{q}\right], x_{1}\right], \ldots\right], x_{n}\right]} \\
& \left.=\sum_{\sigma_{1}} \varepsilon\left(\sigma_{1}\right)\left[\left[\ldots\left[M_{p},\left[\left[\ldots\left[M_{q}, x_{\sigma_{1}(1)}\right], \ldots\right], x_{\sigma_{1}(q)}\right]\right], x_{\sigma_{1}(q+1)}\right], \ldots\right], x_{\sigma_{1}(n)}\right] \\
& \left.\quad+\sum_{\sigma_{2}} \varepsilon\left(\sigma_{2}\right)\left[\left[\ldots\left[M_{q},\left[\left[\ldots\left[M_{p}, x_{\sigma_{2}(1)}\right], \ldots\right], x_{\sigma_{2}(p)}\right]\right], x_{\sigma_{2}(p+1)}\right], \ldots\right], x_{\sigma_{2}(n)}\right]
\end{aligned}
$$

où $\sigma_{1}$ (resp. $\left.\sigma_{2}\right)$ est un “( $\left.q, n-q\right)$-shuffle” (resp. “( $\left.p, n-p\right)$-shuffle”).

Ce lemme se démontre par une itération de l'identité de Jacobi graduée et de la propriété d'antisymétrie du grand crochet.

Comme $\left[M_{p}, M_{q}\right]=\left[M_{q}, M_{p}\right]$, on a la relation

$$
\begin{aligned}
& {\left[\left[\ldots\left[\left[M^{(n+1)}, x_{1}\right], \ldots\right], x_{n}\right]\right.} \\
& \quad=\sum_{p+q=n+1}\left[\left[\ldots\left[\left[M_{p}, M_{q}\right], x_{1}\right], \ldots\right], x_{n}\right] \\
& \left.\quad=2 \sum_{p+q=n+1} \sum_{\sigma} \varepsilon(\sigma)\left[\left[\ldots\left[M_{p},\left[\left[\ldots\left[M_{q}, x_{\sigma(1)}\right], \ldots\right], x_{\sigma(q)}\right]\right], x_{\sigma(q+1)}\right], \ldots\right], x_{\sigma(n)}\right],
\end{aligned}
$$


où $\sigma$ est un " $(q, n-q)$-shuffle".

Le résultat suivant relie les jacobiateurs des crochets $\{\cdot, \ldots, \cdot\}_{M}$ et $\{\cdot, \ldots, \cdot\}_{M}^{*}$ avec les crochets $n$-aires associés aux composantes d'ordre $k$ du carré de $M, M^{(k)}, k=$ $1,2, \ldots, 2(2 m+3)$. En effet, adoptant les notations de [21], désignons par $J_{M}^{n}$ le $n$ ième jacobiateur des crochets $\{\cdot, \ldots, \cdot\}_{M}$ et par $J_{M}^{* n}$ celui des crochets $\{\cdot, \ldots, \cdot\}_{M}^{*}$; soit $\{\cdot, \ldots, \cdot\}_{M^{(k+1)}}$ le crochet $k$-aire associé à la composante d'ordre $k+1$ du carré de $M, k=0,1, \ldots, 2(2 m+3)-1$. On a :

Théorème 2 Soit $\left.M \in \bigwedge^{(2 m+1)} F\right)$; alors :

(1) Le crochet $n$-aire associé à $M^{(n+1)}$ est égal à $2(-1)^{(n+1)} J_{M}^{n}, c^{\prime}$ est-à-dire $\forall x_{1}, \ldots, x_{n} \in$ $\bigwedge F$

$$
\left\{x_{1}, \ldots, x_{n}\right\}_{M^{(n+1)}}=2(-1)^{(n+1)} J_{M}^{n}\left(x_{1}, \ldots, x_{n}\right) .
$$

(2) Le crochet $n$-aire associé à $M^{(2(2 m+3)-(n+1))}$ est égal à $2(-1)^{(n+1)} J_{M}^{* n}$, c'est-à-dire $\forall \alpha_{1}, \ldots, \alpha_{n} \in \bigwedge F^{*}$,

$$
\left\{\alpha_{1}, \ldots, \alpha_{n}\right\}_{M^{(2(2 m+3)-(n+1))}}=2(-1)^{(n+1)} J_{M}^{* n}\left(\alpha_{1}, \ldots, \alpha_{n}\right) .
$$

Démonstration Ce théorème est une conséquence des lemmes 1 et 2; prouvons le point (1). En effet, pour $n=0$, on a :

$$
\begin{aligned}
J_{M}^{0}(1) & =-\left\{\{1\}_{M}\right\}_{M}=-\left\{M_{0}\right\}_{M} \\
& =-\left[M_{1}, M_{0}\right]=-\frac{1}{2} M^{(1)}=-\frac{1}{2}\{1\}_{M^{(1)}} ;
\end{aligned}
$$

d'où

$$
\{1\}_{M^{(1)}}=-2 J_{M}^{0}(1)
$$

Pour $n=1, x \in \bigwedge F$, on a:

$$
\begin{aligned}
J_{M}^{1}(x) & =\left\{\{x\}_{M}\right\}_{M}+\left\{M_{0}, x\right\}_{M} \\
& =\left\{\left[M_{1}, x\right]\right\}_{M}+\left[\left[M_{0}, M_{2}\right], x\right] \\
& =\left[M_{1},\left[M_{1}, x\right]\right]+\left[\left[M_{2}, M_{0}\right], x\right] \\
& =1 / 2\left[\left[M_{1}, M_{1}\right], x\right]+\left[\left[M_{2}, M_{0}\right], x\right] \\
& =1 / 2\left[\left[M_{1}, M_{1}\right]+2\left[M_{2}, M_{0}\right], x\right] \\
& =1 / 2\left[M^{(2)}, x\right]=1 / 2\{x\}_{M^{(2)}} ;
\end{aligned}
$$

d'où

$$
2 J_{M}^{1}=\{\cdot\}_{M^{(2)}} .
$$

Démontrons la relation dans le cas général; pour cela afin d'éviter toute confusion dans la suite, nous noterons le signe de $\operatorname{Koszul}$ par $\varepsilon_{0}(\sigma)$ lorsque les $x_{i}$ sont considérés 
comme éléments de $\bigwedge F$ et nous écrirons tout simplement $\varepsilon(\sigma)$ lorsqu'ils sont considérés comme éléments de $(\bigwedge F)[1]$, c'est-à-dire

$$
\varepsilon_{0}(\sigma)=(-1)^{\sum_{\sigma(i)<\sigma(k)}\left|x_{\sigma(i)}\right|\left|x_{\sigma(k)}\right|}
$$

et

$$
\varepsilon(\sigma)=(-1)^{\sum_{\sigma(i)<\sigma(k)}\left(\left|x_{\sigma(i)}\right|-1\right)\left(\left|x_{\sigma(k)}\right|-1\right)} .
$$

D'après le lemme 2 , pour $x_{1}, \ldots, x_{n} \in \bigwedge F$, on a

$$
\begin{aligned}
& {\left[\left[\ldots\left[M^{(n+1)}, x_{1}\right], \ldots\right], x_{n}\right]=\sum_{p+q=n+1}\left[\left[\ldots\left[\left[M_{p}, M_{q}\right], x_{1}\right], \ldots\right], x_{n}\right]} \\
& \left.\quad=2 \sum_{p+q=n+1} \sum_{\sigma} \varepsilon_{0}(\sigma)\left[\left[\ldots\left[M_{p},\left[\left[\ldots\left[M_{q}, x_{\sigma(1)}\right], \ldots\right], x_{\sigma(q)}\right]\right], x_{\sigma(q+1)}\right], \ldots\right], x_{\sigma(n)}\right] .
\end{aligned}
$$

Revenant à la définition des differents crochets et tenant compte, d'après le lemme 1, du fait que,

$$
\left[\left[\left[\ldots\left[M_{q}, x_{\sigma(1)}\right], x_{\sigma(2)}\right], \ldots\right], x_{\sigma(q)}\right] \in \bigwedge^{2 m+3-q+\sum_{k=1}^{q}\left(\left|x_{\sigma(k)}\right|-1\right)} F
$$

on obtient

$$
\begin{aligned}
& {\left[\left[\ldots\left[M^{(n+1)}, x_{1}\right], \ldots\right], x_{n}\right]} \\
& \quad=2 \sum_{p+q=n+1} \sum_{\sigma} \varepsilon_{0}(\sigma)(-1)^{\theta}(-1)^{\rho}\left\{\left\{x_{\sigma(1)}, \ldots, x_{\sigma(q)}\right\}_{M}, x_{\sigma(q+1)}, \ldots, x_{\sigma(n)}\right\}_{M}
\end{aligned}
$$

où

$$
\theta=\sum_{k=1}^{q-1}(q-k)\left(\left|x_{\sigma(k)}\right|-1\right)
$$

et

$$
\rho=(p-1)\left(-q+\sum_{k=1}^{q}\left(\left|x_{\sigma(k)}\right|-1\right)\right)+\sum_{k=2}^{p-1}(p-k)\left(\left|x_{\sigma(q+k-1)}\right|-1\right) .
$$

Simplifions le produit

$$
\varepsilon_{0}(\sigma)(-1)^{\theta}(-1)^{\rho}(-1)^{\sum_{\sigma(i)<\sigma(k)}\left|x_{\sigma(i)}\right|\left|x_{\sigma(k)}\right|+\theta+\rho} ;
$$


pour cela calculons tout d'abord $\theta+\rho$ sachant que $p+q=n+1$ :

$$
\begin{aligned}
\theta+\rho= & \sum_{k=1}^{q-1}(q-k)\left(\left|x_{\sigma(k)}\right|-1\right)+(p-1)\left(-q+\sum_{k=1}^{q}\left(\left|x_{\sigma(k)}\right|-1\right)\right) \\
& \quad+\sum_{k=2}^{p-1}(p-k)\left(\left|x_{\sigma(q+k-1)}\right|-1\right) \\
= & \sum_{k=1}^{n-1}(n-k)\left(\left|x_{\sigma(k)}\right|-1\right)-(p-1) q \\
= & \sum_{k=1}^{n-1}(n-k)\left(\left|x_{\sigma(k)}\right|-1\right)-p(q+1)+(n+1)
\end{aligned}
$$

d'où

$$
\begin{aligned}
\varepsilon_{0}(\sigma) & (-1)^{\theta}(-1)^{\rho} \\
= & (-1)^{\sum_{\substack{\sigma(i)<\sigma(k) \\
i>k}}\left|x_{\sigma(i)}\right|\left|x_{\sigma(k)}\right|}(-1)^{\sum_{k=1}^{n-1}(n-k)\left(\left|x_{\sigma(k)}\right|-1\right)}(-1)^{(-p(q+1)+(n+1))} \\
= & (-1)^{\sum_{\substack{\sigma(i)<\sigma(k) \\
i>k}}\left|x_{\sigma(i)}\right|\left|x_{\sigma(k)}\right|}(-1)^{\sum_{k=1}^{n-1}(n-k)\left(\left|x_{\sigma(k)}\right|-1\right)}(-1)^{p(q-1)}(-1)^{(n+1)} .
\end{aligned}
$$

Mais, remarquant que dans $\sum_{k=1}^{n-1}(n-k)\left(\left|x_{\sigma(k)}\right|-1\right)$ ne subsistent que les termes où $(n-k)$ est impair, on obtient finalement

$$
\varepsilon_{0}(\sigma)(-1)^{\theta}(-1)^{\rho}=\operatorname{sign}(\sigma) \varepsilon(\sigma)(-1)^{\theta_{n}}(-1)^{n+1}(-1)^{p(q-1)} ;
$$

ce qui nous permet d'avoir

$$
\left[\left[\ldots\left[M^{(n+1)}, x_{1}\right], \ldots\right], x_{n}\right]=2(-1)^{\theta_{n}}(-1)^{n+1} J_{M}^{n}\left(x_{1}, \ldots, x_{n}\right) .
$$

Multipliant les deux membres de cette égalité par $(-1)^{\theta_{n}}$, on obtient

$$
(-1)^{\theta_{n}}\left[\left[\ldots\left[M^{(n+1)}, x_{1}\right], \ldots\right], x_{n}\right]=2(-1)^{n+1} J_{M}^{n}\left(x_{1}, \ldots, x_{n}\right),
$$

c'est-à-dire

$$
\left\{x_{1}, \ldots, x_{n}\right\}_{M^{(n+1)}}=2(-1)^{n+1} J_{M}^{n}\left(x_{1}, \ldots, x_{n}\right) ;
$$

d'où l'égalité désirée.

La démonstration du point 2 du théorème est analogue à celle du point 1 ; d'où le théorème.

Remarque Ce résultat est une application de la version Z Z-graduée du théorème 1 de [21] établi dans le cas $\mathbb{Z}_{2}$-gradué. Ainsi, si $M$ est de carré nul, c'est-à-dire $M^{(k)}=0, \forall k$, les crochets $\{\cdot, \ldots, \cdot\}_{M}$ et $\{\cdot, \ldots, \cdot\}_{M}^{*}$ vérifient les identités de Jacobi généralisées. D’où : 
Corollaire 2 Si $M \in \bigwedge^{(2 m+1)}(F)$ est de carré $n u l$, alors les crochets $\{\cdot, \ldots, \cdot\}_{M}$ (resp. $\left.\{\cdot, \ldots, \cdot\}_{M}^{*}\right)$ définissent sur $(\bigwedge F)[1]\left(\right.$ resp. $\left.\left(\bigwedge F^{*}\right)[1]\right)$ une structure de $L_{\infty}$-algèbre.

Les deux structures définies respectivement sur $(\bigwedge F)[1]$ et $\left(\bigwedge F^{*}\right)[1]$ par les crochets $n$-aires associés à $M$ sont en dualité et on passe de l'une à l'autre grâce au résultat suivant :

Corollaire 3 La correspondance suivante

$$
\begin{aligned}
& L_{M}:\left(\{\cdot, \ldots, \cdot\}_{M}\right) \rightarrow\left(\{\cdot, \ldots, \cdot\}_{M}^{*}\right) \\
& \{\cdot, \ldots, \cdot\}_{M_{n}} \rightarrow\{\cdot, \ldots, \cdot\}_{M_{2 m+3-n}}^{*}
\end{aligned}
$$

entre la collection des crochets $n$-aires définis sur $\bigwedge F$ et celle des crochets $n$-aires définis sur $\bigwedge F^{*}$ est biunivoque et préserve la multilinéarité des crochets $n$-aires associés à $M$.

Pour établir cette correspondance entre les deux collections de crochets, il suffit de remarquer que $\{\cdot, \ldots, \cdot\}_{M_{n}}$ et $\{\cdot, \ldots, \cdot\}_{M_{2 m+3}}^{*}$ s'appliquent respectivement à $n$ éléments de $\bigwedge F$ et à $n$ éléments de $\bigwedge F^{*}$.

Remarque Dans le cas où $[M, M] \neq 0$, comme les crochets $\left[M_{0}, M_{0}\right]$ et

$$
\left[M_{(2 m+3)}, M_{(2 m+3)}\right]
$$

sont tous deux nuls pour cause de degrés, $M$ définit sur $(\bigwedge F)[1]$ et $\left(\bigwedge F^{*}\right)[1]$ des structures de $L_{\infty}$-algèbre d'ordre supérieur à $4 m+4$ au sens de [21].

Remerciements L'auteur tient à remercier Y. Kosmann-Schwarzbach pour ses différentes remarques et suggestions sur le contenu du travail; les remerciements vont également au Centre Abdus Salam ICTP, particulièrement à l'Office of Associate and Federation Schemes, pour l'hospitalité et le soutien tant financier que matériel qui ont permis la réalisation de ce travail.

This work was done within the Associateship Scheme of the Abdus Salam International Centre for Theoretical Physics, Trieste, Italy. Financial support from the Swedish International Development Cooperation Agency (SIDA) is also gratefully acknowledged.

\section{Références}

[1] F. Akman et L. M. Ionescu, Higher derived brackets and deformation theory I. arXiv:math.QA/0504541.

[2] M. Bangoura, Algèbres quasi-Gerstenhaber différentielles. Travaux mathématiques 16(2005), 299-314.

[3] M. De Wilde et P. Lecomte, Formal deformations of the Poisson Lie algebra of a symplectic manifold and star-products. Existence, equivalence, derivations. Nato Adv. Sci. Inst. Ser. C Math. Phys. Sci. 247(1988), 897-960.

[4] V. G. Drinfeld, Hamiltonian structures on Lie groups, Lie bialgebras and the geometric meaning of classical Yang-Baxter equations. (Russian) Dokl. Akad. Nauk SSSR 268(1983), 285-287.

[5] — On almost cocommutative Hopf algebras. Leningrad Math. J. 1(1990), 331-342.

[6] Quasi-Hopf algebras. Leningrad Math. J. 1(1990), 1419-1457. 
[7] J. Huebschmann, Higher homotopies and Maurer-Cartan algebras: quasi-Lie Rinehart, Gerstenhaber, and Batalin-Vilkovisky algebras. Dans: The breadth of symplectic and Poisson geometry, Progr. Math. 232, Birkhäuser, Boston, 2005, 237-302.

[8] Y. Kosmann-Schwarzbach, Grand crochet, crochets de Schouten et cohomologie d'algèbres de Lie. C. R. Acad. Sci. Paris Sér. I Math. 312(1991), 123-126.

[9] Jacobian quasi-bialgebras and quasi-Poisson Lie groups. Contemp. Math. 132(1992), $459-489$.

[10] From Poisson algebras to Gerstenhaber algebras. Ann. Inst. Fourier Grenoble 46(1996), $1243-1274$

[11] — Derived brackets. Lett. Math. Phys. 69(2004), 61-87.

[12] $\longrightarrow$ Quasi, twisted, and all that... in Poisson geometry and Lie algebroid theory. Dans :The breadth of symplectic and Poisson geometry, Progr. Math. 232, Birkhäuser, Boston, 2005, 363-389.

[13] Y. Kosmann-Schwarzbach et F. Magri, Poisson-Nijenhuis structures. Ann. Inst. Henri Poincaré A 53(1990), 35-81.

[14] B. Kostant et S. Sternberg, Symplectic reduction, BRS cohomology, and infinite-dimensional Clifford algebras. Ann. Physics 176(1987), 49-113.

[15] J.-L. Koszul, Crochet de Schouten-Nijenhuis et cohomologie. Astérisque 1985 (numéro hors série), Soc. Math. France, 257-271.

[16] T. Lada et M. Markl, Strongly homotopy Lie algebras. Comm. Algebra 23(1995), 2147-2161.

[17] T. Lada et J. Stasheff, Introduction to SH Lie algebras for physicists. Internat. J. Theoret. Physics 32(1993), 1087-1103.

[18] P. Lecomte et C. Roger, Modules et cohomologie des bigèbres de Lie. C. R. Acad. Sci. Paris Sér. I 310(1990), 405-410.

[19] D. Roytenberg, Courant algebroids, derived brackets and even symplectic supermanifolds. Ph.D. thesis, Berkeley, 1999, mathDG/9910078.

[20] Quasi-Lie bialgebroids and twisted Poisson manifolds. Lett. Math. Physics 61(2002), 123-137.

[21] T. Voronov, Higher derived brackets and homotopy algebras. J. Pure Appl. Algebra 202(2005), 133-153.

[22] $\longrightarrow$ Higher derived brackets for arbitrary derivations. Travaux mathématiques 16(2005), $163-186$.

Département de Mathématiques

Université de Conakry

BP 1147

République de Guinée

email: angoura@gn.refer.org 